\title{
Bilans morphologiques, répartition granulométrique et directions du transport sédimentaire autour du brise-lames de Ste-Anne, delta du Rhône, Méditerranée.
}

\author{
Sabatier François ${ }^{1}$ \\ Provansal Mireille $\mathrm{e}^{\mathrm{let} 2}$ \\ 'Doctorant, CEREGE, Europôle de l'Arbois, B.P.80, 13545 Aix-en- \\ Provence Cedex 04 E-Mail : sabatier@cerege.fr \\ 2 Professeur, Institut de Géographie, Université de Provence, 29 \\ avenue Robert Schuman, 13100 Aix-en-Provence E-Mail : \\ provansal@cerege.fr

\section{Résumé} \\ Le bilan sédimentaire d'un brise-lames construit entre deux épis est analysé \\ par des Modèles Numériques de Terrain. Le trait de côte est stabilisé mais des \\ chenaux d'érosion contre les structures sont mis en évidence. Après une tempête, \\ la répartition granulométrique et la direction du transport sédimentaire (structures \\ sédimentaires et modèle de Gao et Collins) sont étudiés.
}

\section{Abstract}

Sedimentary budget around breakwater and groyne is investiged by Numeric Terrain Model. The Shoreline is stabilized but erosional chanels exists along strucures. A storm event is analysed by several sedimentologic methods: grain size distribution, ripples marks morphology and Gao and Collins model.

\section{Présentation générale}

Le littoral de Ste-Anne, dans le delta du Rhône (figure 1), subit une érosion pouvant atteindre $-10 \mathrm{~m} / \mathrm{an}$ depuis plusieurs décennies (Suanez et Provansal, 1998). Ce secteur est soumis à des houles de SW, SE et S, les houles les plus fortes étant issues du SE (Hmax annuelle $6.2 \mathrm{~m}$ et Tmax annuelle $8 \mathrm{~s}$ ). A la côte, les plans de houles (Sogreah, 1995 et Priand, 1996) décrivent une augmentation des agitations liée à une convergence des orthogonales, en relation avec la présence du prodelta fossile du Vieux Rhône. Le recul du trait de côte met ici en danger la Compagnie des Salins du Midi et des Salines de l'Est (CSMSE) qui exploite les étangs en arrière des plages. La CSMSE a d'abord équipé cette partie du littoral d'une digue frontale pour limiter l'envahissement de l'eau de mer dans les tables salantes (fin des années 70). Cependant, le recul continu du trait de côte à conduit à construire une batterie d'épis de 1988 à 1990. A Ste-Anne, un briselames «test » est construit en 1992 entre deux d'entre eux. Les épis et le briselames sont formés d'un noyau de tout venant (5/50) emballé dans du géotextile et protégé d'une carapace d'enrochement (blocs de 1 à 3 tonnes). L'épis oriental est plus court $(50 \mathrm{~m})$ que l'épi occidental $(65 \mathrm{~m})$. Le brise-lames long de $80 \mathrm{~m}$ est situé à 60 mètres du rivage. La cote d'arase des ouvrages est à $1.5 \mathrm{~m}$ NGF.

Figure 1 : Localisation du site Figure 1 : Site location 
Cet article propose d'abord un bilan sédimentaire pluri-annuel de la plage autour du brise-lames à partir de la superposition de Modèles Numériques de Terrain (MNT). Puis il analyse la répartition granulométrique et la direction du transport sédimentaire lors d'une tempête de SE au moyen d'analyses sédimentologiques.

\section{Bilan morphosédimentaire pluri-annuel autour de l'ouvrage}

\subsection{Morphologie du tombolo et des alvéoles}

La morphologie du tombolo et des alvéoles est décrite d'après un relevé de terrain traité en MNT (théodolite LEICA TC600 et logiciel Autocad/Autosurf®). Le périmètre étudié s'étend de la plage aérienne au brise-lames, à $-2 \mathrm{~m}$ de profondeur environ (figure 2). Derrière le brise-lames s'est formé un tombolo, dont l'axe est décalé par rapport à l'ouvrage de 10-20 mètres vers l'ouest. L'alvéole Ouest comprend une plage de $10 \mathrm{~m}$ environ entre le trait de côte et la digue frontale. Dans l'alvéole Est, la plage varie de 0 à 2 mètres selon les climats de houle. Sur la face interne des épis, des chenaux atteignent -3 à -4 mètres de profondeur, plus creusés le long de l'épi oriental. Sur la face interne du briselames on observe un chenal $\mathrm{E}-\mathrm{W}(-1 \mathrm{~m})$ et deux alvéoles d'érosion $(-2 \mathrm{~m})$. Ces affouillements indiquent la présence de courants érosifs puissants le long des structures. Contre le brise-lames, ils peuvent également être liés au franchissement des lames déferlantes, réalisé pour des houles au large de récurrence annuelle.

Figure 2 : Morphologie des fonds autour des ouvrages.

Figure 2 : Beach morphology and shoreline position around coastal structure

\subsection{Bilan sédimentaire}

La quantification du bilan sédimentaire de 1992 à 1999 est obtenu par superposition diachronique de MNT des plages autour des ouvrages (figure 3). En 1992, le premier MNT, antérieur au brise-lames, est construit depuis deux profils bathymétriques de la CSMSE. A partir de 1996 les MNT sont réalisés par des semi de points denses. Le gain global entre 1992 et 1999 est de $1500 \mathrm{~m} 3$. La comparaison des MNT de novembre 1996 à octobre 1999 (-128 m3) montre une stabilisation du tombolo à l'échelle pluri-annuelle. En 1996-1997, 6 MNT permettent une approche du bilan sédimentaire saisonnier qui apparaît au contraire très contrasté : les houles hivernales favorisent un déficit de sables $(-700 \mathrm{~m} 3)$, celles de beau temps un engraissement $(+750 \mathrm{~m} 3)$.

Figure 3 : Bilan sédimentaire autour des ouvrages.

Figure 3 : Sedimentary budget around coastal structure 


\section{Sédimentologie et direction du transport}

\subsection{Objectifs}

Le fonctionnement hydrosédimentaire autour d'un brise-lames a pu être décrit grâce à des modèles réduits ainsi que des modèles numériques (Sogreah, 1987 ; Nicholson et al., 1997). Par contre, la distribution granulométrique autour des ouvrages reste encore peu étudiée. Elle met en évidence les niveaux d'énergie hydrodynamiques (Bull et al. , 1998) mais ne donne qu'une vision statique des processus. Nous avons donc complété cette méthode d'une approche dynamique par l'analyse des structures sédimentaires et l'utilisation du modèle de Gao et Collins (1991, 1992). Le projet est d'identifier les conséquences d'une tempête sur la granulométrie et les structures sédimentaires à proximité des ouvrages et de tenter d'en déduire les directions de transport.

\subsection{Répartition granulométrique autour de l'ouvrage.}

Les observations et les prélèvements ont été effectués deux jours après une tempête de SE (Hsig 3.2m et Tsig 6s) dans des conditions calmes (Hsig $0.2 \mathrm{~m}$ et Tsig 4s). 167 échantillons ont été prélevés sur la partie superficielle des sédiments $(3-5 \mathrm{~cm})$ selon une maille régulière de $10 \mathrm{~m}$ X $10 \mathrm{~m}$. La granulométrie des sables non décarbonatés a été effectuée mécaniquement sur une colonne de tamis de norme AFNOR. Les indices granulométriques: grain moyen, classement et assymétrie sont calculés en unités phi par la méthode des moments statistiques (Rivière, 1977).

Le grain moyen présente une répartition dissymétrique de part et d'autre du tombolo (figure 4). A l'Est, les sables sont globalement plus fins et leur organisation interne est disparate. A l'Ouest, les sédiments sont plus grossiers et s'organisent en auréoles concentriques de valeurs croissantes depuis le centre de l'alvéole vers la périphérie. Sur le flanc de chacun des deux épis, une bande étroite de sables plus fins apparaît. Le chenal situé sur le flanc interne du briselames est par contre caractérisé par des sables plus grossiers. Le tombolo luimême est hétérogène, il existe un gradient de plus en plus grossier vers le briselames. Avec une répartition moins bien organisée que le grain moyen, les valeurs du classement reflètent aussi une dissymétrie entre les deux alvéoles. A l'Est, les valeurs du classement ne présentent aucune répartition particulière tandis qu'à l'Ouest, on perçoit une organisation concentrique. Les sédiments les mieux classés correspondent aux sables les plus grossiers. Les valeurs d' assymétrie ne présentent aucune organisation particulière. On propose d'expliquer la dissymétrie entre les deux alvéoles par la direction SE des houles de tempêtes qui abordent frontalement l'alvéole Est, alors qu'elles sont diffractées par le brise-lames avant de pénétrer dans l'alvéole Ouest. Dans l'alvéole Est, l'observation lors de la tempête a montré une agitation très forte liée aux interférences entre les houles entrantes et celles réfléchies par la digue frontale. Ces conditions dynamiques élevées justifient le mauvais classement, mais apparaissent curieusement contradictoires avec la finesse relative du matériel sédimentaire. Dans l'alvéole 
Ouest, l'organisation sédimentaire (grain moyen et classement) se calque sur la géométrie des houles déferlantes diffractées par le brise-lames. La bipartition du tombolo coïncide avec la limite atteinte par les eaux lors de la tempête. Au Nord, les sables fins et bien classés correspondent à un stock hérité non submergé lors de l'évènement. Les sables grossiers et mal triés au sud du tombolo correspondent aux croisements des houles diffractées par l'ouvrage. Dans le chenal qui jouxte la face interne du brise-lames la grossièreté des grains et le mauvais classement soulignent des conditions dynamiques élevées liées au franchissement de l'ouvrage. Les deux bandes de sables plus fins contre les épis apparaissent contradictoires avec l'hypothèse généralement admise de courants de retours puissants.

Figure 4 : Répartition spatiale du grain moyen, du classement et de l'asymétrie.

Figure 4: Spatial distribution of mean grain size, Standard Deviation and

\section{Skewness}

\subsection{Analyse des structures sédimentaires.}

Dans la partie immergée, et selon la grille d'échantillonnage, les rides (forme, direction, hauteur et espacements des crêtes) ont été identifiées selon la terminologie de Reineck et Singh (1975) et Leeder (1982). La morphologie décrit l'orientation du déplacement des sables, plus rarement sa direction. L'observation en plongée a permis également de séparer les formes mobiles durant l'échantillonnage, des formes immobiles héritées de la tempête (figure 5). L'orientation des rides mobiles montre des déplacements de direction indéterminée divergents dans l'alvéole ouest et parallèles au tombolo dans la fosse de lévigation de l'alvéole est, où le déferlement des houles de beau temps induit aussi des déplacements dirigés. Les rides immobiles sont situées le long des ouvrages. Les déplacements sont de direction indéterminée sur le flanc du briselames et dirigés vers le large le long des épis (crêtes dissymétriques).

Figure 5 : Direction des courants à partir des rides.

Figure 5 : Current direction based on ripples marks.

\subsection{Direction du transport par la méthode de Gao et Collins}

Le modèle de Gao et Collins $(1991,1992)$ a été testé pour déterminer le sens du transport sédimentaire. Autour du brise-lames de Ste-Anne, cette méthode a aboutit à des résultats incohérents et contradictoires avec l'organisation des structures sédimentaires. Après plusieurs itérations, le calcul basé sur la seule utilisation du grain moyen (transport du fin vers le grossier) fournit les meilleurs résultats (figure 6). Deux cellules de transport correspondant aux deux alvéoles du tombolo sont identifiées. Dans l'alvéole Ouest, les vecteurs de transport sont obliques par rapport à l'épi, puis progressivement diffractés en circulation horaire. Dans l'alvéole Est, les directions reflètent un flux entrant S-N mais sont plus confuses derrière le brise-lames. De part et d'autre du tombolo, le transport s'oriente de la digue frontale vers le brise-lames. En revanche, contre les ouvrages la direction du transport n'a pu être déterminée. Dans les alvéoles, on constate que 
ces vecteurs sont cohérents avec les directions indiquées par les rides mobiles de beau temps observées lors de l'échantillonnage.

Figure 6 Vecteurs de direction à partir du modèle modifié de Gao et Collins

Figure 6 Directionnal vectors based on modified Gao and Collins model

\section{Discussion et conclusion}

Les MNT démontrent la stabilité pluri-annuelle du tombolo engendré par le brise-lames et sa forte variabilité saisonnière. Ils révèlent aussi l'existence de chenaux d'érosion le long des ouvrages. Sur la base de ce constat, on a testé plusieurs méthodes sédimentologiques pour définir les déplacements sédimentaires liés à un événement tempétueux.

La granulométrie met en évidence la dissymétrie de fonctionnement du tombolo et l'existence de différents niveaux d'énergie, plus élevée dans l'alvéole est moins protégée des houles de tempêtes par un épi certainement sousdimensionné. Il reste difficile de donner un sens à la finesse du matériel contre les épis. Nous émettons l'hypothèse d'une décantation du matériel en suspension lié à la période de faible énergie durant l'échantillonnage qui a succédée à la tempête. Les structures sédimentaires démontrent que la partie centrale des alvéoles est constamment remodelée par l'agitation, y compris par des faibles houles de beau temps, alors que les chenaux d'érosion, le long des ouvrages, ont un fonctionnement plus épisodique, lié aux tempêtes. Les vecteurs directionnels décrivent un schéma de circulation, de la digue au brise-lames, conforme aux travaux antérieurs, permettant la construction d'un tombolo (Sogreah, 1987; Nicholson et al., 1997). A proximité des ouvrages, les vecteurs directionnels n'ont pu être déterminés. Ce travail montre donc l'apport et les limites de la méthode de Gao et Collins. Elle est cohérente avec les structures sédimentaires des sables en mouvement au moment des prélèvements. Elle ne rend pas comte de la circulation à proximité des ouvrages, où la structure reste le meilleur indicateur des déplacements induits par les houles de tempêtes.

\section{Remerciements}

Ce travail est réalisé dans le cadre d'une bourse doctorale financée par la Région Provence-Alpes-Côte d'Azur et la Compagnie des Salins du Midi et des Salines de l'Est (CSMSE). Il a reçu une aide financière du Conseil Général des Bouchesdu-Rhône. Les auteurs remercient Mohamed Chaibi et Claude Vella pour leur aide.

\section{Références bibliographiques :}

BRIAND O. et GIEULLES D. (1996) - Analyse d'un ouvrage de bas de plage le bris-lames de St-Anne à Salins de Giraud: un exemple unique en France, Génie Civil Génie Côtier, Dinard, Ecole Pratique des Hautes Etudes et Université du 
Havre, pp. 333-341.

BULL C.F.J., DAVIS A.M. et JONES R. (1998) - The influence of fish-tail groynes (or breakwaters) on the characteristics of the adjacent beach at Llandudno, North Wales, Journal of Coastal Research, 14,1, pp. 93-105.

GAO S. et COLLINS M. (1991) - A critique of the "Mc Laren method" for defining sediment transport paths - discussion, Journal of Sedimentary Petrology, 61, 1, pp.143-146.

GAO S. et COLLINS M. (1992) - Net sediment transport patterns inferred from grain size trends, based upon definition of "transport vector, Sedimentary Geology 80: 47-60.

LEEDER M. R. (1982) - Sedimentology : Process and Product, London, 344p.

NICHOLSON J., BROKER I., ROELVINK J.A., PRICE D., TANGUY J.M. et MORENO L.(1997) - Intercomparaison of coastal areamorphodynamic models, Coastal Engineering, 31, pp. 97-123.

REINECK H. E. and I. B. SINGH (1975) - Depositional sedimentary Environments. New York Heidelberg Berlin, Springer Verlag, 344 p.

RIVIERE A. (1977) - Méthodes granulométriques, techniques et interprétations, Paris, Masson, $170 p$.

SOGREAH (1987) - Ouvrages de protection d'un littoral sableux, Grenoble, pp. 4-65.

SOGREAH (1995) - Etude de l'évolution du littoral sableux de la Camargue, Grenoble, 2 tomes et ann.

SUANEZ S. et PROVANSAL M. (1998) - Large scale evolution of the littoral of the Rhône delta (Southest France), Journal of Coastal Research, 14, 2, pp. 493501 . 


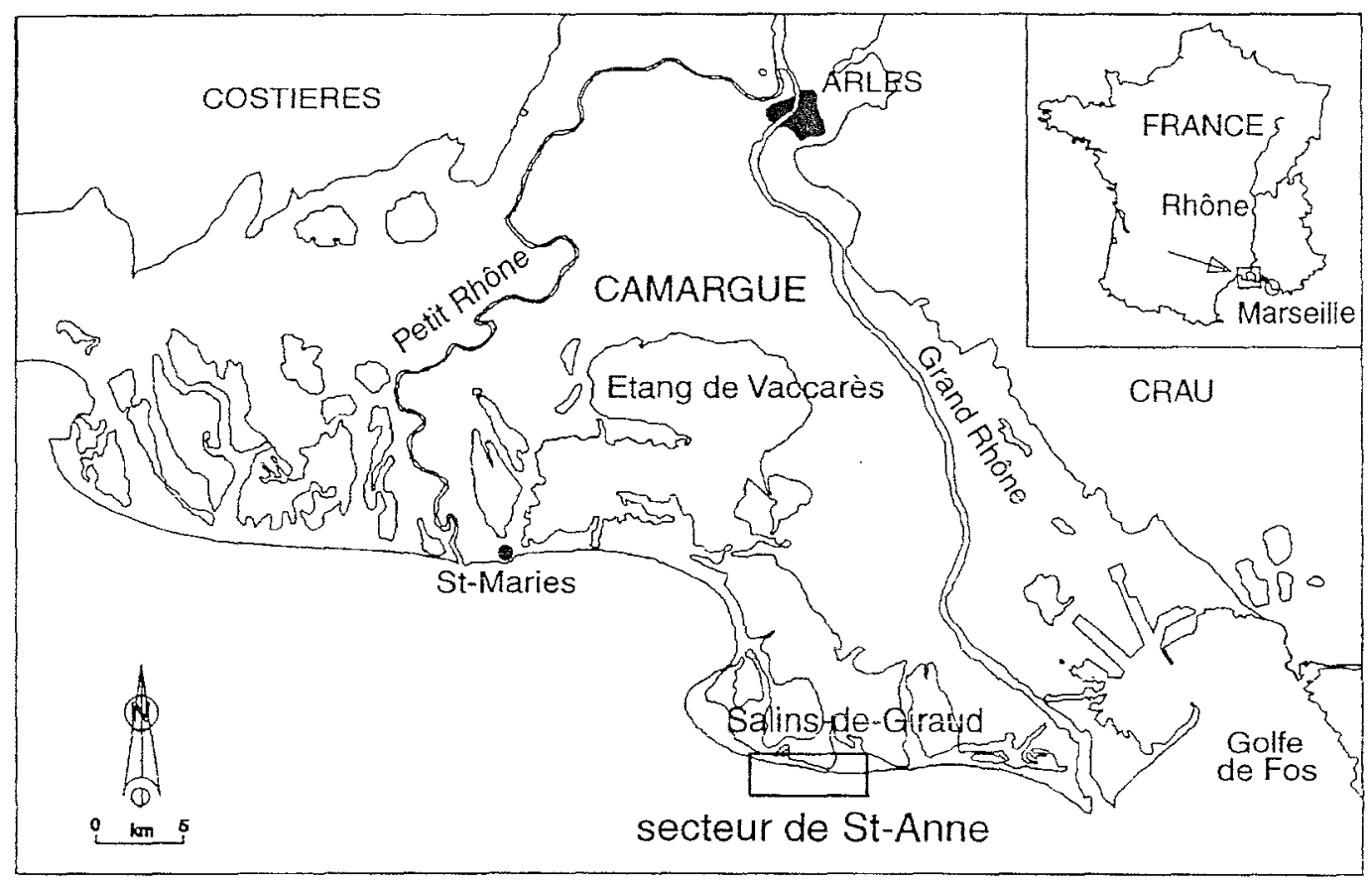

Figure 1 : Localisation du site

Figure 1 : Site location 
digue frontale

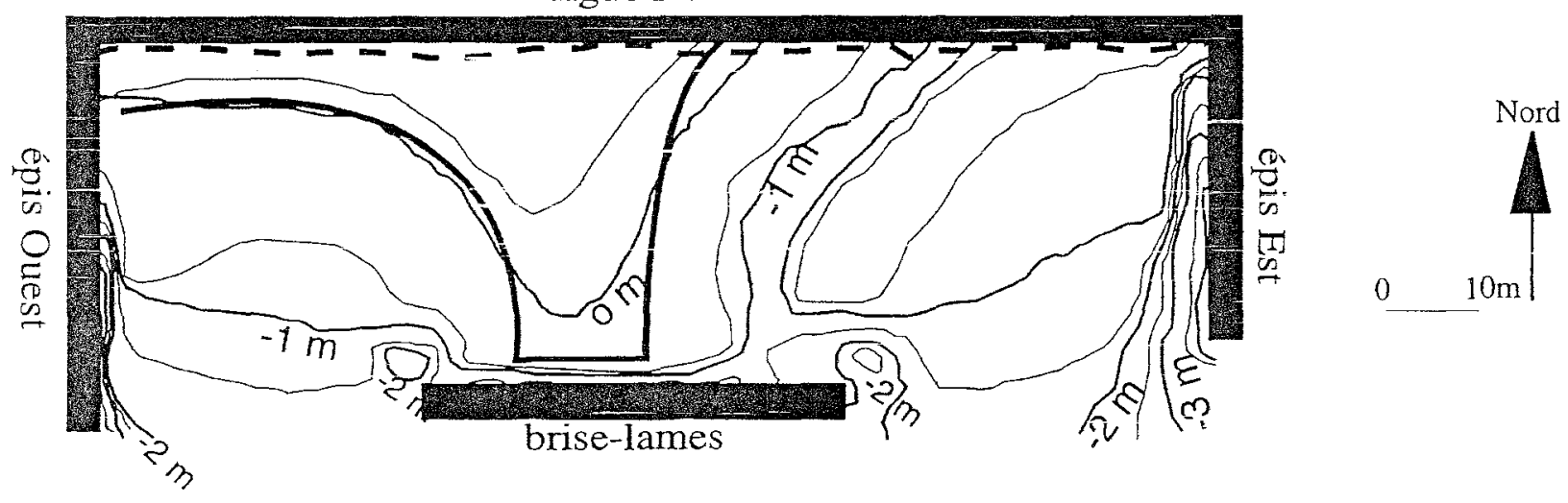

$\longrightarrow$ trait de côte depuis 1993 (approximatif)

- - - trait de côte avant la construction du brise-lames

- courbes de niveau principales (NGF), MNT du 29/10/99

- courbes de niveau secondaires (NGF), MNT du 29/10/99

Figure 2 : Morphologie et différentes positions du trait de côte autour des ouvrages.

Figure 2 : Beach morphology and shoreline position around coastal structure 


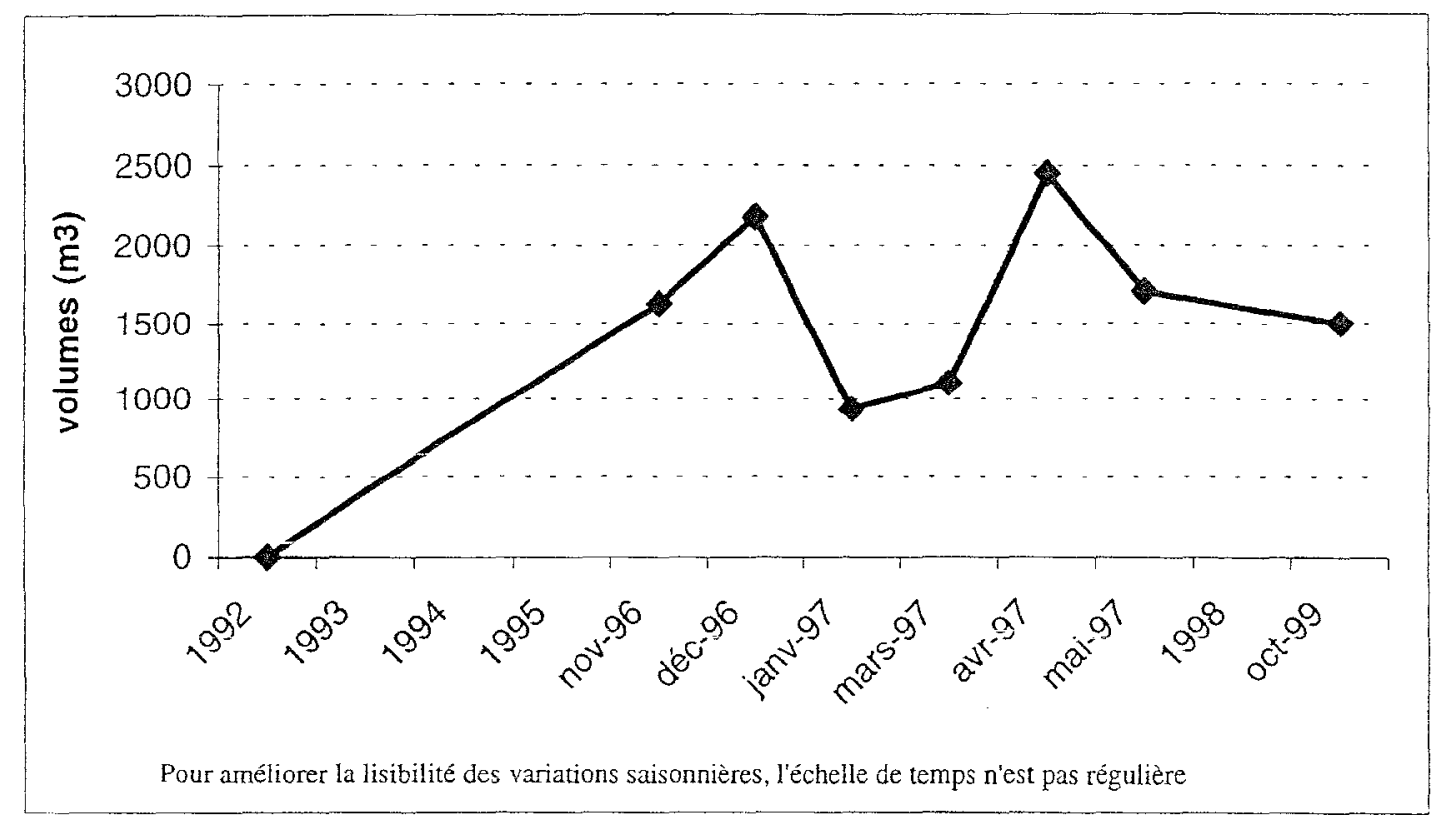

Figure 3 : Bilan sédimentaire autour des ouvrages.

Figure 3 : Sedimentary budget around coastal structure 

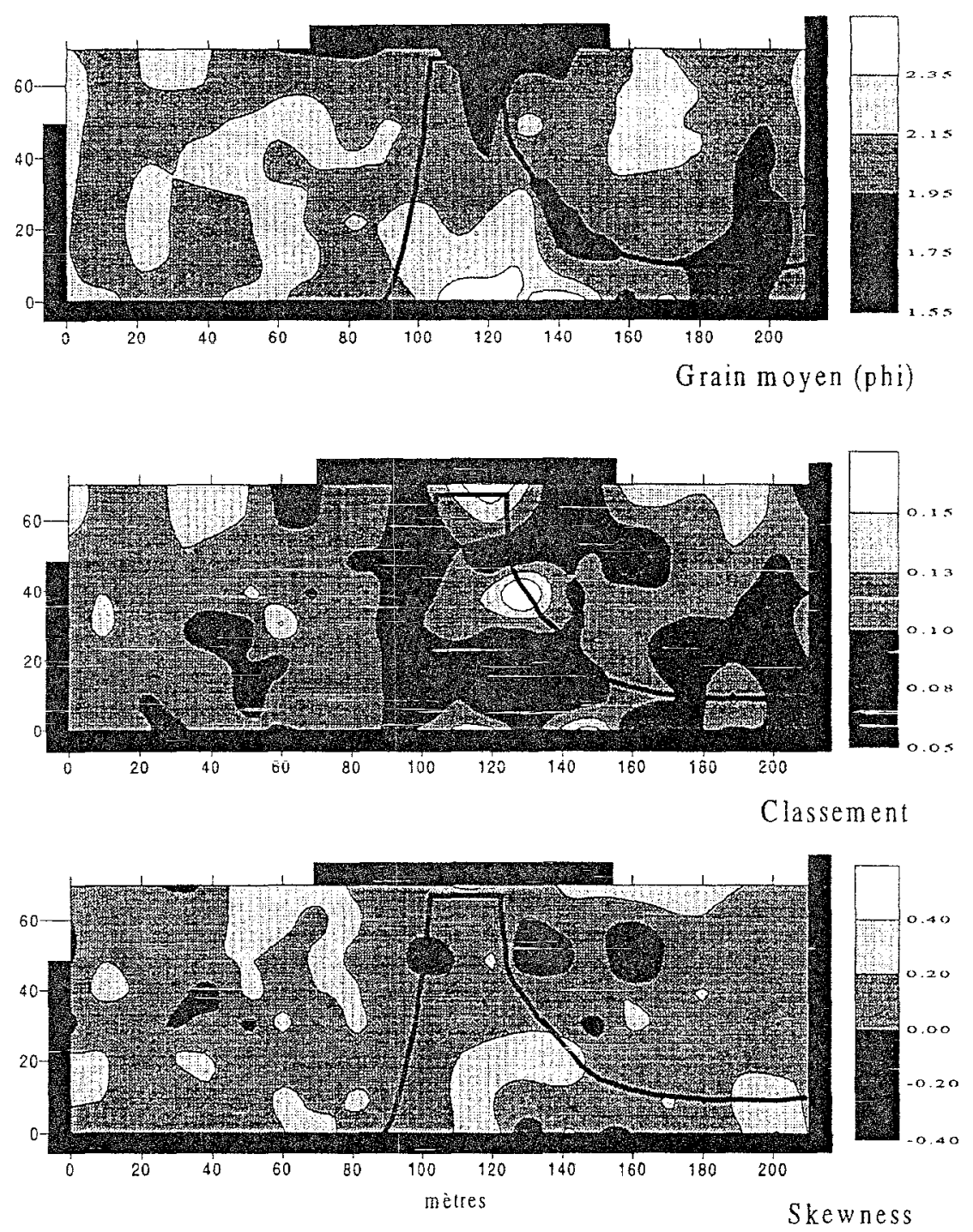

Figure 4 : Répartition spatiale du grain moyen, du classement et de l'assymétrie Figure 4 : Spatial distribution of mean grain size,Standard Deviation and Skewness 


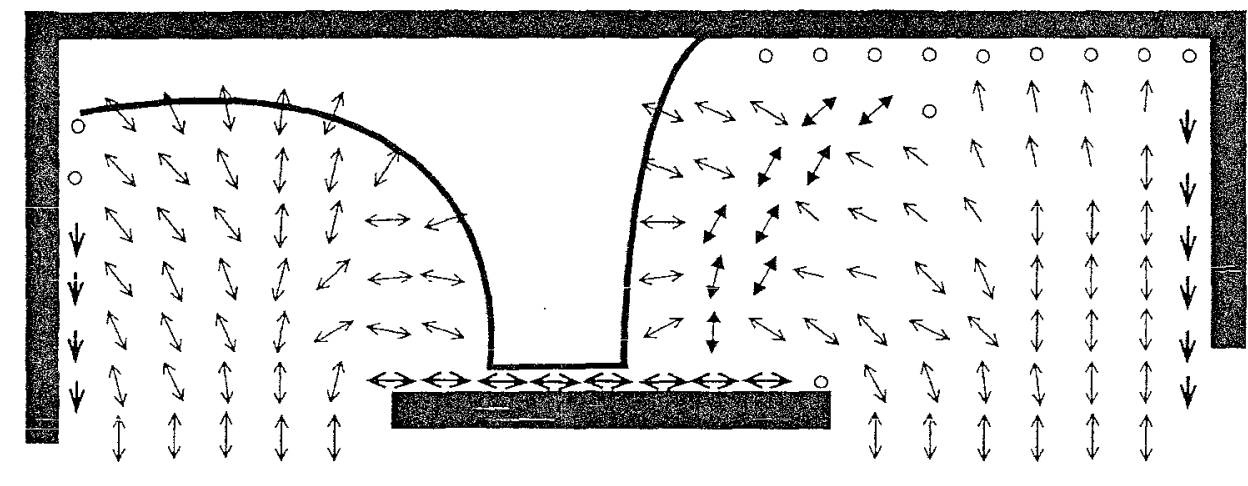

Type I Rides de beau temps

- rides indéterminées

$\leftrightarrow$ I1- Rides de vagues, direction du transport indéterminée.

$\rightarrow$ 12-Rides de courant (déferlement), direction du transport déterminée

$\leftrightarrow$ I3- Rides de courant, direction du transport indéterminée

Type II Rides liées aux tempêtes

$\rightarrow \quad$ II 1 - Rides de courant, direction du transport déterminée

$\leftrightarrow$ I2-Rides de courant, +direction du transport indéterminée.

Figure 5 : Direction des courants à partir des rides.

Figure 5 : Current direction based on ripples marks. 


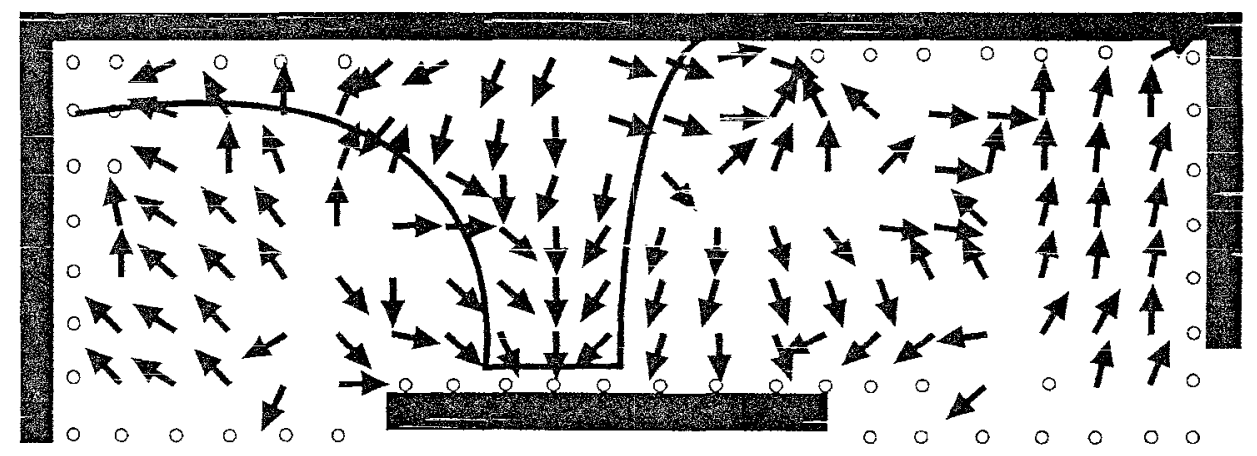

- vecteur de direction calculé à partir du grain moyen

- direction indéterminée

Figure 6 Vecteurs de direction à partir du modèle modifié de Gao et Collins Figure 6 Directionnal vectors based on modified Gao and Collins model 